\title{
Clinical management guidelines of pediatric septic shock
}

\section{Praveen Khilnani}

Septic shock in children is the prototype combination of hypovolemia,cardiogenic and distributive shock. Recently published American college of critical care medinie(ACCM )recommendations for hemodynamic support of neonatal and pediatric patients with sepsis,Surviving sepsis campaign and its pediatric considerations and subsequent revision of definitions for pediatric sepsis has led to compilation of this review article. Practical application of this information in Indian set up in a child with septic shock will be discussed based on available evidence.Though guidelines mainly apply to pediatric age group,however a reference has been made to neonatal age group wherever applicable.

Key Words: Pediatric, sepsis, septic shock, critically ill, management guidelines

\section{Introduction}

\section{Surviving Sepsis Campaign Guidelines for Manage-} ment of Severe Sepsis in Children

In 2003, critical care and infectious disease experts representing 11 international organizations developed management guidelines for other supportive therapies in sepsis, which would be of practical use for the bedside clinician, under the auspices of the Surviving Sepsis Campaign, an international effort to increase awareness and to improve outcome in severe sepsis. ${ }^{[1]}$ The process included a modified Delphi method, a consensus conference, several subsequent smaller meetings of subgroups and key individuals, teleconferences, and electronic-based discussion among subgroups and among the entire committee. Pediatric representatives attended the various section meetings and workshops to contrast adult and pediatric management.

Surviving sepsis campaign guidelines are now published for adults, ${ }^{[1]}$ with pediatric considerations, ${ }^{[2]}$ as a

\section{From:}

Apollo Center for Advanced Pediatrics, IP Apollo Hospital, New Delhi, India.

\section{Correspondence:}

Dr. Praveen Khilnani, Apollo Center for Advanced Pediatrics, Indraprastha Apollo Hospital, New Delhi 110044, India. E-mail: praveenk@nde.vsnl.net.in small section based on the available evidence.

Guidelines of hemodynamic support for pediatrics are also published. ${ }^{[3]}$ Practical application of this information in an Indian setup in a child with septic shock will be discussed. In 1992, the ACCP/SCCM consensus guidelines for definitions of sepsis were published by Bone et al. ${ }^{[4]}$ Definitions have also been recently revised for pediatrics recently, as follows. ${ }^{[5]}$

\section{Definitions}

Hypovolemia is the most common cause of pediatric shock. ${ }^{[5]}$

Septic shock is the prototype combination of hypovolemia and cardiogenic and distributive shock. Following are the latest definitions published in 2005, related to sepsis and septic shock.

\section{Systemic Inflammatory Response Syndrome}

At least two of the following four criteria are present, one of which must be abnormal temperature or leukocyte count.

1. Core (b) temperature of more than $38.5^{\circ} \mathrm{C}$ or less than $36^{\circ} \mathrm{C}$.

\section{Free full text available from www.ijccm.org}


2. Tachycardia, defined as the mean heart rate of more than 2 SD above normal for age in the absence of external stimulus, chronic drugs, or painful stimuli. Otherwise, unexplained persistent elevation over a 0.5-4 $\mathrm{h}$ time period or bradycardia for children aged less than 1 year, defined as the mean heart rate.

3. Less than $10 \%$ for age in the absence of external vagal stimulus, â-blocker drugs, or congenital heart disease, or otherwise, unexplained, persistent depression over a $0.5 \mathrm{~h}$ time period.

4. Mean respiratory rate of more than $2 \mathrm{SD}$ above normal for age or mechanical ventilation for an acute process not related to underlying neuromuscular disease or the receipt of general anesthesia.

5. Leukocyte count elevated or depressed for age (not secondary to chemotherapy-induced leukopenia) or more than $10 \%$ immature neutrophils.

\section{Infection}

A suspected or proven (by positive culture, tissue stain, or polymerase chain reaction test) infection caused by any pathogen or a clinical syndrome associated with a high probability of infection. Evidence of infection includes positive findings on clinical exam, imaging, or laboratory tests (e.g., white blood cells in a normally sterile body fluid, perforated viscus, chest radiograph consistent with pneumonia, petechial or purpuric rash, or purpura fulminans)

\section{Sepsis}

Systemic inflammatory response syndrome in the presence of or as a result of suspected or proven infection.

\section{Severe Sepsis}

Sepsis plus either cardiovascular organ dysfunction or acute respiratory distress syndrome (ARDS), or two or more other organ dysfunctions. Organ dysfunctions are defined in Table 1.

\section{Septic Shock}

Sepsis and cardiovascular organ dysfunction are defined in Table 1. Modifications from the adult definitions are highlighted in boldface. (a) See Table 2 for age-specific ranges for physiologic and laboratory variables, and (b) core temperature must be measured by rectal, bladder, oral, or central catheter probe).

\section{Table 1: Organ dysfunction criteria}

\section{Cardiovascular dysfunction}

Despite administration of isotonic intravenous fluid bolus of more than $40 \mathrm{ml} / \mathrm{kg}$ in $1 \mathrm{~h}$.

Decrease in BP (hypotension) less than $5 \%$ for age or systolic BP of more than 2 SD below normal for age $a$; need for vasoactive drug to maintain BP in normal range (dopamine of more than 5 $\mathrm{mcg} / \mathrm{kg} / \mathrm{min}$ or dobutamine, epinephrine, or norepinephrine at any dose) or two of the following:

- Unexplained metabolic acidosis: base deficit of more than 5.0 $\mathrm{mEq} / \mathrm{l}$.

- Increased arterial lactate of more than two times upper limit of normal.

- Oliguria: urine output of less than $0.5 \mathrm{ml} / \mathrm{kg} / \mathrm{h}$.

- Prolonged capillary refill of more than $5 \mathrm{~s}$.

- Core-to-peripheral temperature gap of more than $3^{\circ} \mathrm{C}$.

\section{Respiratory b}

$\mathrm{PaO}_{2} / \mathrm{FIO}_{2}$ less than 300 in absence of cyanotic heart disease or pre-existing lung disease, $\mathrm{PaCO}_{2}$ of more than 65 torr or $20 \mathrm{mmHg}$ over baseline $\mathrm{PaCO}_{2}$, proven need $c$ or of more than $50 \% \mathrm{FIO}_{2}$ to maintain saturation of more than $92 \%$, or the need for nonelective invasive or noninvasive mechanical ventilation $d$

\section{Neurologic}

Glasgow Coma score of less than 11 or acute change in mental status with a decrease in Glasgow Coma Score of more than three points from abnormal baseline

\section{Hematologic}

Platelet count of $80000 / \mathrm{mm}^{3}$ or a decline of $50 \%$ in platelet count from highest value recorded over the past 3 days (for chronichematology or oncology patients) or international normalized ratio of more than 2

\section{Renal}

Serum creatinine of more than two times upper limit of normal for age or twofold increase in baseline creatinine

\section{Hepatic}

Total bilirubin of more than $4 \mathrm{mg} / \mathrm{dl}$ (not applicable for newborn), ALT 2 times upper limit of normal for age (BP, blood pressure; ALT, alanine transaminase)

a-see Table 2. $b$-ARDS must include a $\mathrm{PaO} / \mathrm{FIO}$ ratio of less than 200 $\mathrm{mm} \mathrm{Hg}$, bilateral infiltrates, acute onset, and no eviđence of left heart failure. Acute lung injury is defined identically, except that the $\mathrm{PaO} / \mathrm{FIO}$ ratio must be less than $300 \mathrm{mmHg}$. $c$ - Proven need assumes oxygen ${ }^{2}$ requirrement was tested by decreasing flow with subsequent increase in flow, if required. $d$-In postoperative patients, this requirement can be met if the patient has developed an acute inflammatory or infectious process in the lungs, which prevents him or her from being extubated.

The detection of altered organ function in the acutely ill patient constitutes multiple organ dysfunction syndrome (two or more organ involvement). The terminology dysfunction identifies this process as a phenomenon in which organ function is not capable of maintaining homeostasis. This process, which may be absolute or relative, can be more readily identified as a continuum of change over time. 
Table 2: Age-specific vital signs and laboratory values

\begin{tabular}{lll}
\hline Age group & $\begin{array}{l}\text { Heart rate } \\
\text { (tachycardia) }\end{array}$ & $\begin{array}{l}\text { Respiratory rate } \\
\text { (bradycardia) }\end{array}$ \\
$0-1$ & $>180$ & $<100$ \\
1 week to 1 month & $>180$ & $<100$ \\
1 month to 1 year & $>180$ & $<90$ \\
$2-5$ years & $>140 \mathrm{NA}$ & $>22$ \\
$6-12$ years & $>130 \mathrm{NA}$ & $>18$ \\
$13-<18$ years & $>110$ & $\mathrm{NA}$
\end{tabular}

$13-<18$ years

$>110$

Leukocyte count
(breaths $/ \min \times 1000 / \mathrm{m}^{3}$ )
$>50$
$>40$
$>34$
$>15.5$ or $<6$
$>13.5$ or $<4.5$
$>14$

SBP

$>34$

$>19,5$ or $<5$

$>17.5$ or $<5$

$<94$

$<105$

$>11$ or $<4,5<117$

Lower values for heart rate, leukocyte count, systolic blood pressure (SBP) are for the $5 \%$ and upper values for heart rate, respiratory rate, or leukocyte count for the $95 \%$.

\section{Rapid Cardiopulmonary Assessment and Clinical Ex- amination of a Patient in Shock}

The most effective and sensitive physiological monitoring available is repeated and careful physical examination was done by an experienced and competent observer. Because the shock can be rapidly fatal, the child must be assessed immediately and comprehensively. In a healthy child the cardiovascular system has remarkable compensatory capability, so there is generally a stability of blood pressure and only an increase in pulse until there is sudden decompensation, which may lead to precipitous cardiac arrest.

In clinical examination, one must note the following points very carefully:

\section{Mental status}

Restless, agitated, anxious, progressive lethargy.

\section{Skin}

Temperature, color, turgor, peticheal rash may be present in meningococcemia or disseminated intravascular coagulation.

\section{Cardiovascular}

By far, the most significant physical findings in septic shock results from autonomic responses to stress. In children, tachycardia occurs early. The younger the child, the more dependent is the cardiac output on the heart rate rather than on increase in stroke volume.

Alteration in blood pressure is a late manifestation of hypovolemia in children, occurring faster in children. Diastolic blood pressure begins to fall early as vascular tone begins to decrease. Systolic blood pressure is well maintained initially and only begins to fall once hemodynamic compromise is severe. Decreasing blood pressure signifies decompensated stage of shock.

In warm phase of septic shock, capillary refill time may be normal; however, signs of hyperdynamic circulation, widened pulse pressure, and hyperdynamic apex beat are important signs. Capillary refill time of more than $5 \mathrm{~s}$ is always abnormal.

\section{Respiratory}

Respiratory rate is increased to compensate for metabolic acidosis. Second, if ARDS is developing, progressive worsening of respiratory distress may occur .

Urine output

Oliguria is common, leading to anuria. It is important to remember that physical findings will vary according to the stage of shock.

\section{Emergency management}

Management of child with septic shock is best started by aggressive goal-directed management in the emergency department.

The treatment of septic shock in children is aimed at optimizing perfusion of critical vascular beds and preventing or correcting metabolic abnormalities arising owing to cellular hypoperfusion. The ultimate goals are to prevent or reverse the defects in cellular substrate delivery and metabolism and to support the patient until homoeostasis is restored.

For all forms of shock, treating the underlying cause is mandatory and speed is essential. Delays in making the diagnosis and initiating treatment (fluid resuscitation as well as appropriate antibiotics), as well as suboptimal resuscitation, contribute to the developments of peripheral vascular failure and irreversible defects in oxygen 
use, which can culminate in vital organ dysfunction.

\section{Priorities of Treatment}

Two major priorities in treatment of septic shock aregiven below.

1. Rapid assessment of patient's disease process.

2. Acheivement of cardiopulmonary stability.

VIP approach can be used in initial treatment of shock, in which "V" stands for ventilation, "l" for infusion, and "P" for pumping or cardiovascular support.

Initial resuscitation of a child in shock involves assessment of airway, administration of oxygen, and establishment of intravenous access.

\section{Airway and Respiratory Support (Ventilation)}

The first priority is to secure the airway and ensure adequate oxygenation and ventilation. High-flow oxygen system (e.g., Venturi masks) must be used. Oxygen supply is optimized by maintaining arterial oxygen saturation, by correcting anemia, and by increasing cardiac output and systemic blood flow. If airway is unstable and adequate oxygenation and ventilation is not achieved, do endotracheal intubation and provide mechanical ventilation. Because mechanical ventilation abolishes or minimizes the work of breathing, reduces oxygen consumption, and improves oxygenation, early respiratory support benefits patients with severe shock in addition to those with ARDS or pulmonary edema.

\section{Cardiovascular Support}

Tissue blood flow must be restored by achieving and maintaining an adequate cardiac output and by ensuring that systematic blood pressure is sufficient to maintain perfusion of vital organs. Cardiovascular support involves manipulation of heart rate and rhythm and of three determinants of stroke volume (preload, myocardial contractility, and afterload).

\section{Rate and Rhythm}

Assuring adequate heart rate and rhythm is the basic need for life support. Monitoring of heart rate is essential in guiding therapy. It is important to keep in mind that heart rate varies according to age and are acceptable within a wide range of normal for age. That includes correction of hypoxia, acidosis, and electrolyte disturbances.

\section{Preload and volume replacement}

Fluid therapy by peripheral, intraosseous, or central venous access should be initiated after adequate control of airway and breathing have been accomplished.

Preload optimization is the most efficient way of increasing cardiac output. Rapid intravascular volume expansion guided by repeated clinical examination and urine output is frequently adequate to restore blood pressure and peripheral perfusion.

Pulmonary edema with volume overload is rare in child patients. Volume replacement of $10-20 \mathrm{ml} / \mathrm{kg}$ with isotonic solutions such as normal saline or ringers lactate can be safely given and repeated if necessary (typically, 40-80 $\mathrm{ml} / \mathrm{kg}$ may be required). Controversy continues about whether colloids or crystalloids are preferable..$^{[5-8]}$ At present, a judicious mixture of crystalloids, blood products to maintain hemoglobin and clotting factors, and colloids to maintain colloid oncotic pressure seems most appropriate and reasonable. As well as being fundamental to the management of hypovolemic shock, replacement of circulating volume is important in managing patients with distributive shock.

The absolute contraindication of preload augmentation in children is a persistent elevation in ventricular filling pressures without an increase in cardiac output. Further preload augmentation does not improve peripheral perfusion and by increasing venous pressure may increase vascular leak, leading to increased tissue edema, most notably pulmonary edema. Chest X-ray (showing enlarged heart shadow and pulmonary edema) or bedside echocardiography are useful adjuncts to clinical examination to identify cardiac decompensation.

\section{Choice of Fluid for Volume Replacement Blood}

To maintain hemoglobin at around $10 \mathrm{~g}$.

\section{Crystalloids}

Cheap, convenient to use, free of side-effects. Rapidly distributed across intravascular and interstitial 
spaces. A volume of two to four times of colloid required for same volume expansion, transient volume expansion.

\section{Colloids}

Colloids such as starch and gelatin produce greater and more sustained increase in plasma volume. Fresh, frozen plasma supplies clotting factors.

\section{Albumin}

Should be used only in special circumstances, e.g., burns and septic shock. The cost of therapy is an issue while considering colloid solutions for expansion of plasma volume.

\section{Inotropic and Vasoactive Agents}

Sepsis-induced myocardial depression is well documented. Before cardiac output and perfusion pressure are restored with drugs, electrolyte abnormalities (such as ionized hypocalcemia) that might impair cardiac performances should be corrected. Metabolic acidosis secondary to tissue hypoxia should be managed by treating the cause. Sodium bicarbonate should be given only for severe acidosis that fails to respond to adequate resuscitation.

If signs of shock persist despite adequate volume replacement and perfusion of vital organs is jeopardized, inotropic drugs may be used to improve cardiac output. ${ }^{\text {[9- }}$ 11] The effects of a particular drug in an individual patient are unpredictable and must be closely monitored. Drugs commonly used in pediatric ICU to increase myocardial contractility include the following:

\section{Dopamine}

It has a, â, and dopaminergic (ä) actions that are dosedependent. At low doses ( $<3 \mathrm{mcg} / \mathrm{kg} / \mathrm{min}$ ), it primarily causes weak renal and splanchnic vasodilatation, and at $3-10 \mathrm{mcg} / \mathrm{kg} / \mathrm{min}$ it exerts a positive myocardial inotropic efect. At higher doses (>10 $\mathrm{mcg} / \mathrm{kg} / \mathrm{min}$ ), it has strong vasoconstricting a-effect, in addition to positive inotropic effect. The so-called "renal dose" of dopamine (2-5 $\mathrm{mcg} / \mathrm{kg} / \mathrm{min}$ ) for renal vasolidation has been overemphasized and is of less practical significance in clinical setting. The primary indication for dopamine is the need to increase myocardial contractility after preload restoration. The usual dose is $5-20 \mathrm{mcg} / \mathrm{kg} / \mathrm{min}$ titrated to desired effect. Dopamine (in doses of more than 5 $\mathrm{mcg} / \mathrm{kg} / \mathrm{min}$ ) should preferably be given via central line to prevent ischemic necrosis of the skin.

\section{Dobutamine}

It is a selective $\beta_{1}$ agonist. It causes an increase in cardiac contractility and reduces peripheral resistance. The reduction in afterload and improved myocardial performance lowers ventricular filling pressures. The usual dose is $5-20 \mathrm{mcg} / \mathrm{kg} / \mathrm{min}$. It should not be used alone in septic shock owing to the risk of further drop in blood pressure. Dopamine or adrenaline can be used to prevent hypotension owing to vasoconstrictive effect.

\section{Adrenaline (epinephrine)}

It is an a- and â-adrenergic agonist. It is used in situations in which dominant hemodynamic feature is peripheral vascular failure, as in septic shock. At higher doses severe vasoconstriction can lead to lactic acidosis and renal and splanchnic ischemia. The usual dose is $0.1-1$ $\mathrm{mcg} / \mathrm{kg} / \mathrm{min}$. It should be titrated closely and the minimum dose should be used as required.

\section{Noradrenaline (Norepinephrine)}

It is an a- and â-agonist (a-effect being greater than âeffect). Cardiac contractility is increased but also causes massive increase in myocardial oxygen consumption and afterload; so cardiac output may not actually increase. The usual dose is $0.05-1 \mathrm{mcg} / \mathrm{kg} / \mathrm{min}$. In severe septic shock with hypotension despite the use of adrenaline secondary to intense vasodilatation, noradrenaline may be useful in increasing peripheral vascular resistance to improve blood pressure.

\section{Vasopressin}

In severe warm shock with hypotension resistant to noradrenaline, vasopressin may be tried.

\section{Afterload reduction}

Caution should be used in using afterload reduction indiscriminately in septic shock without simultaneous inotropic support. Both nitroprusside and nitroglycerin lower systemic vascular resistance in children and are useful afterload-reducing agents. These agents act via the generation of nitric oxide. Nitroprusside has potent peripheral arterial vasodilating effects. Nitroglycerin is a more potent venodilator and pulmonary vasodilator. 
Close monitoring and volume augmentation are frequently required when vasodilators are used to decrease pulmonary vascular resistance.

Amrinone and milrinone are newer inotropic agents with properties of afterload reduction and myocardial diastolic relaxation (lusotropic effect). ${ }^{[10-12]}$ Milrinone is commonly used for cardiogenic shock, which is frequently associated with septic shock.

Early goal directed therapy helps keep the cost and duration of hospital stay to a minimum. The following section describes the current consensus on management of sepsis in children, based on available evidence. ${ }^{[2]}$. These guidelines mainly apply to the pediatric age group. These guidelines may not be applicable to the neonatal age group, though occasional reference has been made to the neonatal situations.

\section{Need for Early Intubation and Ventilation}

Owing to low functional residual capacity, young in fants and neonates with severe sepsis may require early intubation. ${ }^{[13]}$ Unfortunately, no objective clinical criteria specific to pediatric septic shock for timing of endotracheal intubation (other than the standard indications, which include shock) exist in literature. Therefore, it is reasonable to consider endotracheal intubation when shock is persistent even after a volume resuscitation of more than 40-60 ml/kg. Children with sepsis requiring aggressive fluid resuscitation frequently have worsening tachypnea and increasing oxygen requirement, clinically depicting early ARDS. These patients will require early intubation and mechanical ventilation. The principles of lung-protective strategies (low tidal volumes and permissive hypercapnea) are applied to children as they are to adults. In premature infants, additional attention is paid to avoiding hyperoxemia to prevent retinopathy.

\section{Fluid Resuscitation}

Intravenous access for fluid resuscitation and inotrope/ vasopressor infusion is more difficult to attain in children than in adults. The American Heart Association has well-established pediatric advanced life support guidelines for emergency establishment of intravascular support, including intraosseous access. ${ }^{[14]}$ On the basis of many studies, it is accepted that aggressive fluid resuscitation with crystalloids or colloids is of fundamental importance to survival of septic shock in children. ${ }^{[8,15]}$
There is only one randomized, controlled trial comparing the use of colloid with crystalloid resuscitation (dextran, gelatin, lactated Ringers, or saline) in children with dengue shock. ${ }^{[8]}$ All these children survived, regardless of the fluid used, but the longest time to recovery from shock occurred in children who received lactated Ringers. Among patients with the narrowest pulse pressure, there was a suggestion that colloids were more effective than crystalloids in restoring normal pulse pressure. Fluid infusion is best initiated with boluses of $20 \mathrm{ml} / \mathrm{kg}$ over 5-10 min and titrated to clinical monitors of cardiac output, including heart rate, urine output, capillary refill, and level of consciousness. A $60-\mathrm{ml}$ syringe filled with fluid drawn via the fluid bag with a three-way connection can be conveniently used to push fluid boluses in the absence of a volumetric pump.

Children normally have a lower blood pressure than adults and can prevent reduction in blood pressure by vasoconstriction and increasing heart rate. Therefore, blood pressure by itself is not a reliable endpoint for assessing the adequacy of resuscitation. However, once hypotension occurs, cardiovascular collapse may soon follow.

Hepatomegaly occurs in children who are fluid-overloaded and can be a helpful sign of the adequacy of fluid resuscitation. Other practical ways to assess fluid overload are jugular venous distension, heart size, and pulmonary congestion on chest X-ray. Gold standard still remains the measurement of a central venous pressure.

Large fluid deficits typically exist, and initial volume resuscitation usually requires $40-60 \mathrm{ml} / \mathrm{kg}$ but can be much higher. ${ }^{[9,15,16]}$ As a word of caution in neonates, the use of aggressive fluid therapy may be limited by patency of ductus arteriosus, risk of intraventricular hemorrhage, and right heart failure owing to pulmonary hypertension.

\section{Vasopressors/inotropes}

These should only be used after appropriate volume resuscitation. Children with severe sepsis can present with low cardiac output and high systemic vascular resistance (cold shock, more common scenario), high cardiac output and low systemic vascular resistance, or low cardiac output and low systemic vascular resistance shock. Early inotropic support should be started in the case of fluid-refractory shock or a life-threatening hypo- 
tension when fluid bolus has been initiated. Dopamine is the first choice of support for the pediatric patient with hypotension refractory to fluid resuscitation. The choice of vasoactive agent is determined by the clinical examination. Dopamine-refractory shock may reverse with epinephrine (adrenaline) or norepinephrine (noradrenaline) infusion. ${ }^{[16]}$ Pediatric patients with low cardiac output states may benefit from use of dobutamine. The use of vasodilators can reverse shock in pediatric patients who remain hemodynamically unstable with a high systemic vascular resistance state, despite fluid resuscitation and implementation of inotropic support. ${ }^{[9,16]}$ Nitrosovasodilators with a very short half-life (nitroprusside or nitroglycerin) are used as first-line therapy for children with epinephrine-resistant low cardiac output and elevated systemic vascular-resistance shock. Inhaled nitric oxide reduced extracorporeal membrane oxygenation use when given to the term neonates with persistent pulmonary artery hypertension of the newborn and sepsis in a randomized, controlled trial. ${ }^{[17]}$ When pediatric patients remain in a normotensive, low-cardiacoutput, and high-vascular-resistance state, despite epinephrine and nitrosovasodilator therapy, the use of a phosphodiesterase inhibitor should be strongly considered, such as milrinone. ${ }^{[12,18,19]}$ Vasopressin therapy should be considered in warm shock unresponsive to fluid and norepinephrine.

\section{Early Antibiotics}

After appropriate cultures are taken, the early use of a broad-spectrum systemic antimicrobial therapy based on clinical suspicion is reasonable, although no randomized studies exist in children. Adult data support the use of early appropriate antibiotics to impact favorably on morbidity from septic shock.

\section{Therapeutic Endpoints}

Therapeutic endpoints are capillary refill of less than 2 $\mathrm{s}$, normal pulses with no differential between peripheral and central pulses, warm limbs, urine output of more than $1 \mathrm{ml} / \mathrm{kg} / \mathrm{h}$, normal mental status, decreased lactate, and increased base deficit and superior vena cava or mixed venous oxygen saturation of more than $70 \%$. When employing measurements to assist in identifying acceptable cardiac output in children with systemic arterial hypoxemia such as cyanotic congenital heart disease or severe pulmonary disease, the arterial-venous oxygen content difference is a better marker than mixed venous hemoglobin saturation with oxygen. Optimizing preload optimizes cardiac index. As noted above, blood pressure by itself is not a reliable endpoint for resuscitation. Rarely, if a pulmonary artery catheter is utilized, therapeutic endpoints are cardiac index of more than 3.3 and less than $6.0 \mathrm{l} / \mathrm{m} / \mathrm{m}^{2}$ with normal perfusion pressure (mean arterial pressure minus central venous pressure) for age. Use of pulmonary artery catheter has declined over the years owing to no well-demonstrated therapeutic benefit in patients with septic shock. Table 3 shows a flow diagram summarizing an approach to pediatric septic shock. ${ }^{[3]}$

\section{Electrolyte Balance}

An attempt should be made to check and correct common electrolyte problems related to sodium (hyponatremia), potassium, and ionized calcium (ionized hypocalcemia).

\section{Steroids}

Hydrocortisone therapy should be reserved for use in children with catecholamine resistance and suspected or proven adrenal insufficiency. Patients at risk include children with severe septic shock and purpura, ${ }^{[20,21]}$ children who have previously received steroid therapies for chronic illness, and children with pituitary or adrenal abnormalities. There are no strict definitions, but adrenal insufficiency in the case of catecholamine-resistant septic shock is assumed at a random total cortisol level of less than $18 \mu \mathrm{g} / \mathrm{dl}(496 \mathrm{nM} / \mathrm{l})$. There is no clear con-

Table 3: ACCM recommendations for neonatal and pediatric septic shock management ${ }^{[3]}$

Normal BP, cold shock SVC $\mathrm{O}_{2}$ saturation less than 70

Low BP, cold shock, SVC $\mathrm{O}_{2}$ saturation less than 70

Low BP, warm shock

\section{Recognize mental status,poor perfusion}

Maintain airway,establish access, push $20 \mathrm{ml} / \mathrm{kg}$ up to $60 \mathrm{ml} / \mathrm{kg}$ fluid. Observe in picu if positive response Recognize fluid-refractory shock, start central line, dopamine, establish arterial monitoring if fluidrefractory dopamine-resistant shock (10 $\mathrm{mcg} / \mathrm{kg} / \mathrm{min})$. Start epinephrine for cold, norepinephrine for warm shock. If risk of adrenal insufficiency (38-39), give hydrocortisone.

Add vasodilator, consider volume

Titrate volume and epinephrine

Give norepinephrine, fluid, consider vasopressin 
sensus for the role of steroids or best dose of steroids in children with septic shock. A post-30-min or -60-min adrenocorticotropic hormone stimulation test increase in cortisol of $\leq 9 \mu \mathrm{g} / \mathrm{dl}(248 \mathrm{nM} / \mathrm{l})$ also makes that diagnosis. There are two randomized, controlled trials that used shock dose hydrocortisone (25 times higher than the stress dose) in children, both in dengue fever. The results were conflicting. ${ }^{[22,23]}$ Dose recommendations vary from 1 to $2 \mathrm{mg} / \mathrm{kg}$ for stress coverage (based on clinical diagnosis of adrenal insufficiency) to $50 \mathrm{mg} / \mathrm{kg}$ for empirical therapy of shock followed by the same dose as a 24-h infusion. Thus, the dose of steroids remains controversial.

\section{Conclusions}

Pediatric recommendations for management of severe sepsis in children include a more likely need for endotracheal intubation and mechanical ventilation owing to low functional residual capacity. Infants and children are recognized to have more difficult intravenous access, therefore necessitating the use of intraosseous access, as required. Early fluid resuscitation based on weight with $40-60 \mathrm{ml} / \mathrm{kg}$ or higher may be needed.

Decreased cardiac output and increased systemic vascular resistance tends to be the most common hemodynamic profile. Dopamine is recommended as the initial agent for hemodynamic support.Pediatric recommendations include greater use of physical examination therapeutic endpoints.

The issue of high-dose steroids for therapy of septic shock remains unsettled, although recommendation include use of steroids for catecholamine-unresponsive shock in presence of a suspected or proven adrenal insufficiency.

There is greater risk of hypoglycemia with aggressive glucose control.

\section{References}

1. Dellinger RP, Carlet JM, Masur H, Gerlach H, Calandra T, Cohen $\mathrm{J}$, et al. Surviving sepsis campaign guidelines for management of severe sepsis and septic shock. Crit Care Med 2004;32:858-73.

2. Parker MM, Hazelzet JA, Carcillo JA. Pediatric considerations. Crit Care Med 2004;32:591-4.

3. Carcillo JA, Fields Al. Task Force Committee members: clinical practice parameters for hemodynamic support of pediatric and neonatal patients in septic shock. Crit Care Med 2002;30:136578.

4. Bone RC, Balk RA, Cerra FB, et al. Definitions for sepsis and organ failure and guidelines for the use of innovative therapies in sepsis. Chest 1992;101:1644-55.

5. Goldstein B, Giroir B, Randolph A, et al. International pediatric sepsis consensus conference: definitions for sepsis and organ dysfunction in pediatrics. Pediatr Crit Care Med 2005;6:2-8.

6. Griffel MI, Kaufman BS. Pharmacology of colloids and crystalloids. Critical Care Clinics 1992;8: 235-53.

7. Ranjit S, Kisson N, Jayakumar I. Aggressive management of dengue shock syndrome may decrease mortality rate: a suggested protocol. Pediatr Crit Care Med 2005;6:412-9

8. Ngo NT, Cao XT, Kneen R, Wills B, Nguyen VM, Nguyen TQ, et al. Acute management of dengue shock syndrome: A randomized double-blind comparison of 4 intravenous fluid regimens in the first hour. Clin Infect Dis 2001;32:204-13.

9. Ceneviva G, Paschall JA, Maffei F, Carcillo JA. Hemodynamic support in fluid-refractory pediatric septic shock. Pediatrics 1998;102:e19.

10. Barton P, Garcia J, Kouatli A, et al. Hemodynamic effects of i.v. milrinone lactate in pediatric patients with septic shock: a prospective, double-blinded, randomized, placebo-controlled, interventional study. Chest 1996;109:1302-12

11. Lindsay CA, Barton $P$, Lawless $S$, et al. Pharmacokinetics and pharmacodynamics of milrinone lactate in pediatric patients with septic shock. J Pediatr 1998;132:329-34.

12. Irazuzta JE, Pretzlaff RK, Rowin ME. Amrinone in pediatric refractory septic shock: an open-label pharmacodynamic study. Pediatr Crit Care Med 2001;2:24-8

13. Pollard AJ, Britto J, Nadel S, DeMunter C, Habibi P, Levin M. Emergency management of meningococcal disease. Arch Dis Child 1999;80:290-6.

14. Kanter RK, Zimmerman JJ, Strauss RH, Stoeckel KA. Pediatric emergency intravenous access: evaluation of a protocol. $A m ~ J$ Dis Child 1986;140:132-4

15. Carcillo JA, Davis AL, Zaritsky A. Role of early fluid resuscitation in pediatric septic shock. JAMA 1991;266:1242-5.

16. Powell KR, Sugarman LI, Eskenazi AE, Woodin KA, Kays MA, McCormick KL, et al. Normalization of plasma arginine vasopressin concentrations when children with meningitis are given maintenance plus replacement fluid therapy. J Pediatr 1991;117:515-22.

17. Keeley SR, Bohn DJ. The use of inotropic and afterload-reducing agents in neonates. Clin Perinatol 1988;15:467-89.

18. Roberts JD, Jr, Fineman JR, Morin FC, III. Inhaled nitric oxide and persistent pulmonary hypertension of the new born. $N$ Engl J Med 1997;336:605-10. 
19. Barton P, Garcia J, Kouatli A, Kitchen L, Zorka A, Lindsay C, et al. Hemodynamic effects of i.v. milrinone lactate in pediatric patients with septic shock: a prospective, double-blinded, randomized, placebo-controlled, interventional study. Chest 1996;109:1302-12.

20. De Kleijn ED, Joosten KF, Van Rijn B, Westerterp M, De Groot $\mathrm{R}$, Hokken-Koelega AC, et al. Low serum cortisol in combination with high adrenocorticotrophic hormone concentrations are associated with poor outcome in children with severe meningococcal disease. Pediatr Infect Dis J 2002;21:330-6.
21. Riordan FA, Thomson AP, Ratcliffe JM, Sills JA, Diver MJ, Hart CA. Admission cortisol and adrenocorticotrophic hormone levels in children with meningococcal disease: evidence of adrenal insufficiency? Crit Care Med 1999;27:2257-61.

22. Min M, U T, Aye M, Shwe TN, Swe T. Hydrocortisone in the management of dengue shock syndrome. Southeast Asian J Trop Med Public Health 1975;6:573-9.

23. Sumarmo, Talogo W, Asrin A, Isnuhandojo B, Sahudi A. Failure of hydrocortisone to affect outcome in dengue shock syndrome. Pediatrics 1982;69:45-9. 